\title{
Comparative phylogenomics of Streptococcus pneumoniae isolated from invasive disease and nasopharyngeal carriage from West Africans
}

\author{
Eric S Donkor ${ }^{1,5}$, Richard A Stabler ${ }^{1}$, Jason Hinds ${ }^{3}$, Richard A Adegbola ${ }^{4}$, Martin Antonio ${ }^{2}$ and Brendan W Wren ${ }^{1 *}$
}

\begin{abstract}
Background: We applied comparative phylogenomics (whole genome comparisons of microbes using DNA microarrays combined with Bayesian-based phylogenies) to investigate S. pneumoniae isolates from West Africa, with the aim of providing insights into the pathogenicity and other features related to the biology of the organism. The strains investigated comprised a well defined collection of 58 invasive and carriage isolates that were sequenced typed and included eight different $S$. pneumoniae serotypes $(1,3,5,6 \mathrm{~A}, 11,14,19 \mathrm{~F}$ and $23 \mathrm{~F})$ of varying invasive disease potential.
\end{abstract}

Results: The core genome of the isolates was estimated to be $38 \%$ and was mainly represented by gene functional categories associated with housekeeping functions. Comparison of the gene content of invasive and carriage isolates identified at least eleven potential genes that may be important in virulence including surface proteins, transport proteins, transcription factors and hypothetical proteins. Thirteen accessory regions (ARs) were also identified and did not show any loci association with the eleven virulence genes. Intraclonal diversity (isolates of the same serotype and MLST but expressing different patterns of ARs) was observed among some clones including ST 1233 (serotype 5), ST 3404 (serotype 5) and ST 3321 (serotype 14). A constructed phylogenetic tree of the isolates showed a high level of heterogeneity consistent with the frequent $\mathrm{S}$. pneumoniae recombination. Despite this, a homogeneous clustering of all the serotype 1 strains was observed.

Conclusions: Comparative phylogenomics of invasive and carriage S. pneumoniae isolates identified a number of putative virulence determinants that may be important in the progression of $S$. pneumoniae from the carriage phase to invasive disease. Virulence determinants that contribute to S. pneumoniae pathogenicity are likely to be distributed randomly throughout its genome rather than being clustered in dedicated loci or islands. Compared to other S. pneumoniae serotypes, serotype 1 appears most genetically uniform.

\section{Background}

Streptococcus pneumoniae is part of the normal bacterial flora of the upper respiratory tract, but is also associated with severe invasive diseases, including meningitis, pneumonia and septicaemia as well as non-invasive diseases such as otitis media [1]. Transmission of S. pneumoniae occurs through respiratory droplets and is more commonly associated with healthy individuals who carry the organism in the upper respiratory tract $[2,3]$. Worldwide, the annual incidence of invasive pneumococcal

\footnotetext{
* Correspondence: Brendan.Wren@lshtm.ac.uk

'Department of Pathogen Molecular Biology, London School of Hygiene and Tropical Medicine, London WC1E 7HT, UK

Full list of author information is available at the end of the article
}

disease (IPD) is about one million and though a global problem, the public health impact of IPD is higher in the developing world, where children less than 5 years of age are most affected [4,5].

The capsule is considered the main virulence determinant of S. pneumoniae, and only a few capsular types tend to be associated with invasive disease which is partly due to differential ability of the variant capsular types to resist phagocytosis [6,7]. Epidemiological evidence indicates that while some capsular types are often associated with invasive disease, some may be associated with carriage, while others are associated with both invasive disease and carriage [8-12]. In addition to the capsule, it is known that other pathogenic factors are 
required by $S$. pneumoniae for virulence [13], but the genetic factors that explain the pathogenesis and virulence of the organism is not fully understood.

Comparative whole genome analysis using DNA microarrays has been utilised to investigate several bacterial pathogens. The approach involves assessing the absence or presence of genes from strains based on reference genome(s) fixed to microarray, followed by robust statistical algorithms to infer the evolutionary relationships between test strains that is usually represented as a phylogenetic tree [14-18]. This allows interrogation of the genome content of bacterial strains from a variety of sources, environments and disease states, and the identification of genetic markers that may explain how different strains are adapted to their respective niches or disease capability. Few comparative genomics studies have been carried out on S. pneumoniae, and these studies were based mainly on strains from developed countries and none from SubSaharan Africa [19-23], where the organism exacts its greatest toll. Though these studies have contributed significantly to our understanding of S. pneumoniae, several aspects of the organism particularly, its pathogenicity, evolution and population structure in the Sub-Saharan Africa is still inadequately understood. In view of this, we carried out comparative phylogenomics (whole genome comparisons of microbes using DNA microarrays combined with Bayesian-based phylogenies) of $58 \mathrm{~S}$. pneumoniae epidemiologically well defined isolates from West Africa with the aim of providing insights into the pathogenicity and other features related to the biology of the organism.

\section{Results and discussion}

\section{Strain selection}

A total of 58 isolates were used in this study, and were collected from three West African countries including The Gambia (52), Nigeria (4) and Ghana (2). All isolates were serotyped [24] and multilocus sequence typed [25] (Figure 1). The isolates comprised 35 invasive and 23 carriage isolates and were recovered from subjects of an age range of 3 months to 58 years. The carriage isolates were recovered from the nasopharynx of healthy human populations $[9,26]$, while invasive isolates were recovered from specimens of blood (87\%), CSF (10\%) and lung and knee aspirates $(3 \%)$ of patients with IPD $[8,27,28]$. Based on information from capsule serotype, the isolates were selected to cover pneumococcal serotypes of varying invasive disease potential in West Africa [8,9,26-29]. Eight serotypes were selected and included serotypes 1, 3, 5, 6A, 11, 14, $19 \mathrm{~F}$ and $23 \mathrm{~F}$ (Table 1). In West Africa, Serotypes 1 and 5 are common in IPD but rare in carriage and represent serotypes of high invasive disease potential; serotypes 3, 11 and $19 \mathrm{~F}$ are common in carriage but not in invasive disease, and represent serotypes of low invasive disease potential; serotypes 6A, 14, and $23 \mathrm{~F}$ are common in both invasive disease and carriage, and represent serotypes of intermediate disease potential. Overall, the isolates studied covered 35 different sequence types; invasive isolates covered 22 sequence types while the carriage isolates covered 16 sequence types.

\section{Core gene set of S. pneumoniae strains}

Whole genome microarray comparisons of 58 isolates of S. pneumoniae were used to compute the minimal core gene set. This was achieved by calculating the total number of coding sequences (CDSs) that had a GACK (Genome Analysis by Charlie Kim) score of 'present' in every isolate and the control strain (TIGR4) using the advanced filtering function available in Genespring 6.1. The minimal core gene set for the $S$. pneumoniae isolates was $831 \mathrm{CDSs}$, which translates to $38 \%$ of the total genome of the isolates. Similarly, individual core gene sets were computed for invasive and carriage isolates and were found to be $1162 \mathrm{CDSs}$ (84\%) and 919 CDSs $(63 \%)$ respectively $(\mathrm{p}<0.05)$. The low core genome estimate of $38 \%$ observed in this study is quite similar to a core genome of $46 \%$ reported by Hiller et al. [22] but contrast significantly with a core genome of $73 \%$ reported by Obert et al. [21] and $80 \%$ reported by Tettelin et al. [20]. However, Hiller et al. [22] demonstrated that individual strains core orthologous clusters account for $68-79 \%$ of the genome. Reported core gene sets of some other streptococci species include $58 \%$ for S. thermophilus [30], 82.5\% for S. uberis [31] and $82 \%$ for S. agalactiae [32]. Relatively low core gene of $28 \%$ has been reported for some non-streptococcal organisms such as Yersinia enterocolitica [16]. Thus core genome quantification may vary significantly among different bacterial strain collections and is highly dependent on the cut off method used as well as the core genome definition. The relatively low core genome reported in this study may reflect the more stringent approach used to compute the core genome (Section "Microarray data analysis and comparative phylogenomics").

As expected, the $S$. pneumoniae core gene set was represented by many of the functional categories that are involved in housekeeping functions such as DNA metabolism, intermediary metabolism, protein synthesis and cellular processes. This concurs with other S. pneumoniae microarray studies [20-22]. Conserved housekeeping genes including those identified in the core genome of the West African isolates have been shown to be abundant in sequenced pneumococcal genomes [33-37]. Comparison of eight nasopharyngeal S. pneumoniae genomes with nine published genomes (including TIGR4 and R6) identified 1,454/3,170 (46\% ) orthologous gene clusters conserved among all 17 strains [22]. The core genes consisted mainly of housekeeping genes but also contained 462 hypothetical proteins with no 


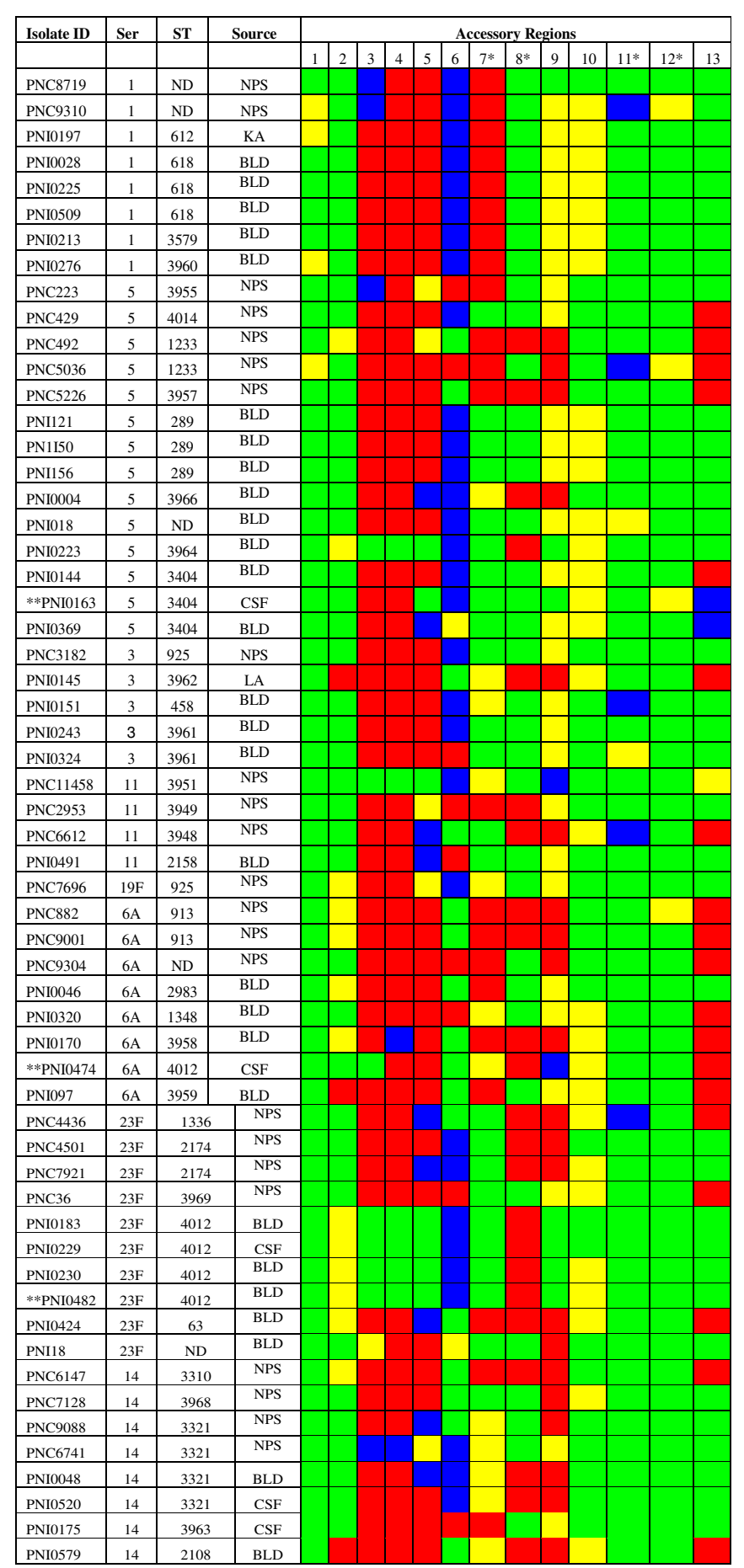

Green colour- AR is completely present

Red colour- AR is completely absent

Yellow- most genes of AR $(>50 \%)$ are present

Blue- most genes of $\mathrm{AR}(>50 \%)$ are abse

Source: NPS-nasopharyngeal swab; BLD-blood; CSF-cerebrospinal fluid; LA-lung aspirate; KA-knee aspirate

ND- ST not determined

* contain virulence genes identified by signature tagged mutagenesis [41]

I** associated with case fatality

Figure 1 Distribution of accessory regions among S. pneumoniae isolates of different serotypes and sequence types. 
Table 1 Serotype distribution of invasive and carriage $S$. pneumoniae isolates used for comparative phylogenomics analysis

\begin{tabular}{lccc}
\hline Serotype & No. of invasive isolates & No. of carriage isolates & Total \\
\hline 1 & 6 & 2 & 8 \\
\hline 3 & 4 & 1 & 5 \\
\hline 5 & 9 & 5 & 14 \\
\hline $6 \mathrm{~A}$ & 5 & 3 & 8 \\
\hline 11 & 1 & 3 & 4 \\
\hline 14 & 4 & 4 & 8 \\
\hline $19 \mathrm{~F}$ & 0 & 1 & 1 \\
\hline $23 \mathrm{~F}$ & 6 & 4 & 10 \\
\hline Total & $\mathbf{3 5}$ & $\mathbf{2 3}$ & $\mathbf{5 8}$ \\
\hline
\end{tabular}

known function [22]. More than $70 \%$ of the West African S. pneumoniae core genes were present in the core gene set of Hiller et al. [22]. Virulence determinant CDSs including transport proteins and various enzymes such as hyaluronidase, neuraminidase A, phosphoglucomutase and triosephosphate isomerase were identified in the S. pneumoniae core gene set in this study. By comparison, hyaluronidase and neuraminidase A were also demonstrated to be conserved within the 17 genomes analysed by Hiller et al. [22]. The presence of virulence determinants in all the invasive as well as carriage isolates in the current study probably indicates that these virulence determinants are necessary, but not adequate, to determine the ability of an isolate to cause invasive disease. Also, analysis of the core gene set of the isolates showed that a wide range of mobile and extrachromosomal elements were conserved, which agrees generally, with information obtained from pneumococcal genomes that have been fully sequenced [20,33-37]. Within the Hiller et al. core gene list are twelve transposases listed [22], which were also present in the core gene list of our study.

\section{Putative virulence determinants and accessory regions}

Overall, comparison of the gene content of invasive and carriage isolates identified at least eleven CDSs that were significantly associated with invasive isolates compared to carriage isolates (Table 2). IgA protease showed the largest difference between invasive and carriage isolates. This surface protein degrades IgA and thus helps $S$. pneumoniae to evade host immune system and provide an opportunity for more effective invasion [38,39]. Several transport proteins of the $\mathrm{ABC}$ type, were also significantly associated with invasive isolates and may be based on the fact that these transport proteins are involved in the transport of metal ions or nutrients which are required by pathogenic bacteria for growth and metabolic activities [40]. Several transcriptional genes were associated with invasive isolates/disease
Table 2 Genes that showed significant differences between invasive and carriage isolates

\begin{tabular}{lllc}
\hline Gene & \multirow{2}{*}{ Function } & \multicolumn{2}{c}{ Frequency } \\
\cline { 3 - 4 } & & Invasive & Carriage \\
\cline { 3 - 4 } \cline { 3 - 4 } & $\mathbf{( N = 3 5 )}$ & $\mathbf{( N = \mathbf { 2 3 } )}$ \\
\hline SP0071 & immunoglobulin A1 protease & 35 & 2 \\
\hline SP0091 & ABC transporter, permease protein & 18 & 5 \\
\hline SP0161 & hypothetical protein & 25 & 7 \\
\hline SP0238 & hypothetical protein & 25 & 6 \\
\hline SP0491 & hypothetical protein & 26 & 9 \\
\hline SP0514 & hypothetical protein & 25 & 9 \\
\hline SP0743 & transcriptional regulator & 30 & 14 \\
\hline SP0955 & competence protein & 27 & 9 \\
\hline SP1032 & iron-compound ABC transporter & 25 & 5 \\
\hline SP1612 & hypothetical protein & 30 & 12 \\
\hline SP1800 & transcriptional activator & 31 & 14 \\
\hline
\end{tabular}

$\mathrm{N}$ indicates total number of invasive or carriage isolates.

which has been previously reported [41]. Several other proteins were also associated with invasive isolates, but were mainly hypothetical proteins and therefore require further investigation.

An accessory region was defined as three or more contiguous genes not conserved in all the isolates. Thirteen accessory regions (ARs) were identified in this study (Table 3) and the distribution of such regions among the study isolates is presented in Figure 1. By comparison previous studies have reported 13-38 ARs $[21,42,43]$. Nine ARs identified in the current study have been previously reported and include AR2, AR3, AR4, AR5, AR6, AR7, AR8, AR9 and AR13 [21,42,43]. Four ARs including AR1, AR10, AR11 and AR12 identified in this study have not been previously reported and represent novel ARs. In the case of AR1 and AR10, none of the genes in these regions have been associated with virulence and thus their functional role in virulence is not clear. Two of the novel ARs namely, AR11 and AR12 contained genes identified by Signature Tagged Mutagenesis (STM) as required for virulence in mice, but none of these ARs was associated with invasive disease [41]. The poor correlation of invasive isolates or serotypes of high invasive disease potential with ARs that contain virulence genes has also been reported by Bloomberg et al. [42]. In the study of Bloomberg et al. [42], though 24 ARs containing virulence genes were identified, only two of such ARs were preferentially found in invasive isolates or serotypes of high invasive disease potential.

Despite the poor correlation between invasive disease and ARs that contain virulence genes, differences in virulence between different clones of the same serotype could be explained by the distribution of such ARs in some cases (evidence provided below). This indicates that the role of ARs in pneumococcal virulence may be 
Table 3 Accessory regions identified among S. pneumoniae isolates

\begin{tabular}{|c|c|c|}
\hline Accessory Region & TIGR4 locus & Gene annotation \\
\hline \multirow[t]{3}{*}{ AR1 } & SP0482 & hypothetical protein \\
\hline & SP0483 & ABC transporter, ATP-binding protein \\
\hline & SP0484 & hypothetical protein \\
\hline \multirow[t]{3}{*}{ AR2 } & SP1050 & transcriptional regulator, putative \\
\hline & SP1051 & hypothetical protein \\
\hline & SP1052 & phosphoesterase, putative \\
\hline \multirow[t]{3}{*}{ AR3 } & SP1062 & ABC transporter, ATP-binding protein \\
\hline & SP1063 & $A B C-2$ transporter, permease protein, putative \\
\hline & SP1064 & transposase, IS200 family \\
\hline \multirow[t]{3}{*}{ AR4 } & SP1129 & integrase/recombinase, phage integrase family \\
\hline & SP1130 & transcriptional regulator \\
\hline & SP1131 & transcriptional regulator, putative \\
\hline \multirow[t]{4}{*}{ AR5 } & SP1134 & hypothetical protein \\
\hline & SP1135 & hypothetical protein \\
\hline & SP1136 & hypothetical protein \\
\hline & SP1137 & GTP-binding protein, putative \\
\hline \multirow[t]{17}{*}{ AR6 } & SP1315 & V-type ATP synthase subunit D \\
\hline & SP1316 & V-type ATP synthase subunit B \\
\hline & SP1317 & V-type ATP synthase subunit A \\
\hline & SP1318 & V-type ATP synthase subunit F \\
\hline & SP1319 & V-type sodium ATP synthase, subunit C \\
\hline & SP1320 & V-type sodium ATP synthase, subunit E \\
\hline & SP1321 & V-type ATP synthase subunit K \\
\hline & SP1322 & V-type ATP synthase subunit I \\
\hline & SP1323 & hypothetical protein \\
\hline & SP1324 & ROK family protein \\
\hline & SP1325 & oxidoreductase, Gfo/ldh/MocA family \\
\hline & SP1326 & neuraminidase, putative \\
\hline & SP1327 & hypothetical protein \\
\hline & SP1328 & sodium: solute symporter family protein \\
\hline & SP1329 & $\mathrm{N}$-acetylneuraminate lyase \\
\hline & SP1330 & $\mathrm{N}$-acetylmannosamine-6-phosphate 2-epimerase \\
\hline & SP1331 & phosphosugar-binding transcriptional regulator, putative \\
\hline \multirow[t]{4}{*}{ AR7 } & SP1341 & ABC transporter, ATP-binding protein \\
\hline & SP1342 & toxin secretion ABC transporter, ATP-binding/permease protein \\
\hline & SP1343* & prolyl oligopeptidase family protein \\
\hline & SP1344* & hypothetical protein \\
\hline \multirow[t]{4}{*}{ AR8 } & SP1433 & transcriptional regulator, AraC family \\
\hline & SP1434* & ABC transporter, ATP-binding/permease protein \\
\hline & SP1435 & ABC transporter, ATP-binding protein \\
\hline & SP1436 & hypothetical protein \\
\hline \multirow[t]{2}{*}{ AR8 } & SP1437 & hypothetical protein \\
\hline & SP1438 & ABC transporter, ATP-binding protein \\
\hline \multirow[t]{4}{*}{ AR9 } & SP1616 & allulose-6-phosphate 3-epimerase \\
\hline & SP1617 & PTS system, IIC component \\
\hline & SP1618 & PTS system, IIB component \\
\hline & SP1619 & PTS system, IIA component \\
\hline
\end{tabular}


Table 3 Accessory regions identified among S. pneumoniae isolates (Continued)

\begin{tabular}{lll}
\hline & SP1620 & PTS system, nitrogen regulatory component IIA \\
\hline AR10 & SP1621 & putative transcription anti terminator BgIG family protein \\
\hline & SP1622 & transposase, IS200 family \\
\hline SP1738 & guanylate kinase \\
\hline AR11 & hP1739 & hypothetical protein \\
\hline & SP1740 & hypothetical protein \\
\hline SP1856* & transcriptional regulator, MerR family \\
\hline SP1857 & cation efflux system protein \\
\hline SP1858 & transcriptional regulator, TetR family \\
\hline SP1859* & transporter, putative \\
\hline SP1860 & choline transporter \\
\hline SP1896* & sugar ABC transporter, permease protein \\
\hline SP1897 & sugar ABC transporter, sugar-binding protein \\
\hline SP1898* & alpha-galactosidase \\
\hline SP2159 & fucolectin-related protein \\
\hline SP2160 & hypothetical protein \\
\hline SP2161 & PTS system, IID component \\
\hline SP2162 & PTS system, IIC component \\
\hline & PP2163 system, IIB component \\
\hline
\end{tabular}

* contain virulence genes identified by signature tagged mutagenesis [41].

serotype dependent which has also been reported $[21,42]$. Included in this study, were four invasive isolates of the serotype 5 virulent PMEN clone ST 289, and also two serotype 5 carriage isolates of ST 1233 which is considered less virulent. The pattern of AR distribution of the ST 289 isolates was the same and carried all the ARs associated with virulence in this study (ARs 7, 8, 11 and 12). However, the ST 1233 isolates were deficient in three of the four ARs associated with virulence including AR7, AR8 and AR11. Thus these differences in ARs of the two serotype 5 clones may explain the enhanced virulence of ST 289. A similar observation has been reported for two serotype 19 F clones namely, ST 162 which is a virulent clone and ST 425, a non-virulent clone [42]. These observations also highlight the variations in virulence of clones of the same serotype and are important in pneumococcal vaccination, where virulent clones of a serotype rather than non-virulent clones of that serotype, undergo capsular switching and emerge with non-vaccine serotypes $[44,45]$. For an invasive serotype like serotype 5 , it also shows that the ability of an isolate to cause invasive disease is not only dependent on the capsule type but also the genetic background of the strain.

Though ARs may have some relevance in pathogenicity, the extent to which ARs contribute to pneumococcal virulence is still not very clear. In this study, the 13 ARs identified did not show loci association with any of the eleven potential virulence genes identified. Analysis of the distribution of virulence genes identified by Hava and Camilli in TIGR4 indicates that the virulence genes did not cluster [41]. These observations suggest that virulence determinants that contribute to S. pneumoniae pathogenicity are likely to be distributed randomly throughout its genome rather than being clustered in dedicated loci or islands. This agrees with the findings of Obert et al. [21] which showed that ARs are more likely to adapt $S$. pneumoniae to carriage rather than invasive disease. Thus ARs may not play a highly prominent in pathogenicity as observed in pathogens such as uropathogenic Escherichia coli [46].

From Figure 1, it can be observed that some isolates of the same serotype and ST were found to express different patterns of ARs, which can be seen for ST 1233 (serotype 5), ST 3404 (serotype 5) and ST 3321 (serotype 14). This phenomenon of intraclonal diversity has also been observed in studies carried out by Silva et al. [43] and Bloomberg et al. [42], and shows that strains of the same serotype and ST may exhibit genetic and phenotypic differences. In the study by Silva et al. [43] different patterns of ARs was observed among pneumococcal isolates of ST 124 (serotype 14), while Bloomberg et al. [42] observed different AR patterns among isolates of ST 176 (serotype 6B), ST 124 (serotype 14) and ST 156 (serotypes 14 and $19 \mathrm{~F}$ ). Thus the current study provides evidence of the phenomenon of intraclonal diversity beyond clones and serotypes that have been previously reported. Bloomberg et al. [42] pointed out that intraclonal diversity was rare among serotypes of high invasive disease potential, as it was not observed among clones of serotypes 1,4 and $7 \mathrm{~F}$ 
included in their study. This finding contrasts with the current study, where intraclonal diversity was consistently exhibited by clones (ST 3404 and ST 1233) of serotype 5, a serotype of high invasive disease potential. Nevertheless, it can be observed that while intraclonal diversity occurred among several serotype 5 clones, it did not occur among the more virulent ST 289 (serotype 5) PMEN clone, indicating that intraclonal diversity may be relatively rare among more virulent clones. This is also the case for the virulent ST 618 (serotype 1) clone and also the ST 4012 (serotype $23 \mathrm{~F}$ ) clone, which is a novel clone and inferred to be virulent, as it was the most frequent cause of mortality (Figure 1). This suggests some association of these virulent clones with stability (uniform genetic content). Dagerhamn et al. [47] have demonstrated that some pneumococcal accessory regions may predict genetic relatedness similar to that predicted by MLST, which they attributed to the influence of recombination on variations in housekeeping genes (used for MLST) and as well as accessory regions. Data on intraclonal diversity from the current study further suggests that in some cases accessory regions may also provide better resolution than MLST, as highly genetically similar isolates of the same serotype and MLST can be distinguished by their accessory regions patterns. This shows the potential as a pneumococcal typing scheme based on accessory regions which would provide similar results to MLST but of better resolution. However, it should be noted that typing by analysis of ARs could be especially susceptible to being confounded by horizontal gene transfer.

\section{Comparative phylogenomics}

The data obtained from microarray analysis was used to generate a phylogenetic tree which is shown in Figure 2. Three of the isolates (PNI676, PNI0108 and PNC12026) subjected to phylogenomic analysis showed a distant association with all the other isolates. These three isolates were subjected to molecular serotyping using another type of microarray [48] to confirm their identity as $S$. pneumoniae. This showed that the three isolates were not $S$. pneumoniae isolates but likely to be a closely related Streptococcus species such as S. mitis or S. oralis, and hence their separation from S. pneumoniae isolates in the phylogenetic tree, which confirms the credibility of the phylogenetic relationship among the isolates. MLST of the three isolates also showed that the sequences were divergent from those of known MLST alleles. The three non-pneumococcal isolates were excluded in analysis of the core genome (Section "Core gene set of S. pneumoniae strains") as well as analysis of putative virulence determinants and accessory regions (Section "Putative virulence determinants and accessory regions").

Phylogenetic analysis of the S. pneumoniae isolates showed two major clades, with each clade comprising a mixture of invasive and carriage isolates of varied serotypes (Figure 2). Despite the heterogeneous clustering of serotypes, all of the eight serotype 1 isolates (six invasive and two carriage isolates) formed a subclade (Figure 2A). Recently, Donati et al. [23] constructed a phylogenetic tree based on 44 sequenced pneumococcal genomes covering 19 different serotypes and 24 MLST clonal clusters. By comparison, in this study, the poor correlation observed between a serotype of an isolate and its position in the tree except for serotype 1, agrees well with the study by Donati et al. [23]. Similarly, the poor correlation observed between an isolate from an invasive or carriage source and its position in the tree also agrees with the study by Donati et al. [23]. The high level of heterogeneity among isolates in the phylogenetic tree of this study is probably due to recombination which occurs frequently among pneumococci. A recent study by Croucher et al. [49] found more than 700 recombination events in 240 strains of the PMEN1 $\left(\right.$ Spain $^{23 \mathrm{~F}}$-1) multidrug-resistant lineage. According to Feil et al. [50], evolution of the pneumococcal population is dominated by recombination, and can abolish any deep-rooted phylogenetic signal resulting in a pattern of heterogeneity as observed in this study. The homogeneous clustering observed among the serotype 1 isolates agrees with the uniform distribution of ARs observed among the serotype 1 isolates, and reflects the fact that, because this serotype is rarely carried, it is less likely to undergo recombination. Within the phylogenetic tree, clustering of isolates of the same MLST was observed (Figure 2B), which has also been reported by Donati et al. [23] and Dagerhamn et al. [47], and provides evidence of the agreement between microarray and MLST. This implies that the frequent pneumococcal recombination did not eliminate phylogenetic signals related to a common ancestor though it may have weakened such signals.

An attempt was made to use MacClade 4 to identify CDSs which were associated with relevant clades and subclades in the S. pneumoniae phylogenetic tree (Figure 2). The two major clades formed, were associated with presence/absence of AR6, AR8, AR9 and AR13 (Table 3). These ARs have been reported to have some importance in pneumococcal pathogenicity $[21,51,52]$. The fact that each clade comprised a mixture of invasive and carriage isolates probably support the earlier claim in this study that ARs may have little relevance in pneumococcal pathogenicity. The formation of the serotype 1 cluster of isolates (Figure 2A) was associated with $10 \mathrm{CDSs}$, all of which were highly divergent or absent from these isolates.

\section{Conclusions}

The current study is unique in that it is based on a relatively large number (58) of $S$. pneumoniae isolates from 


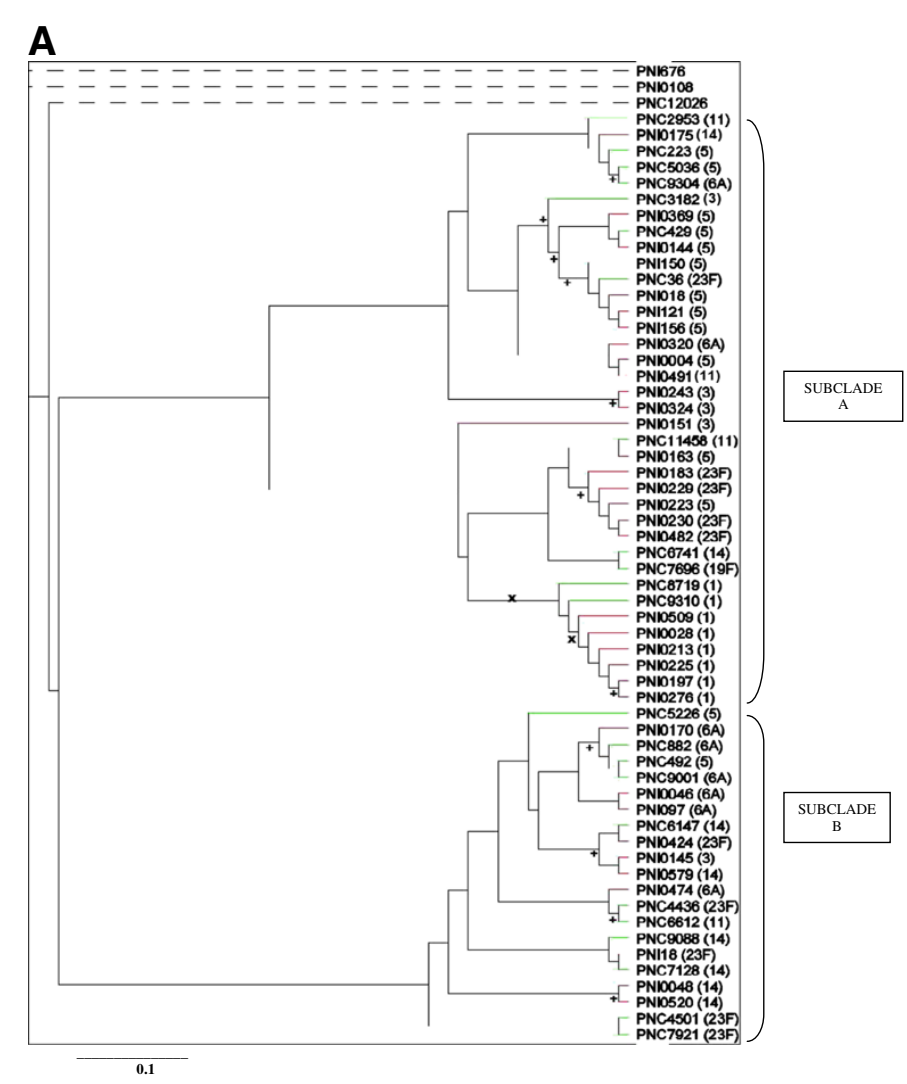

B

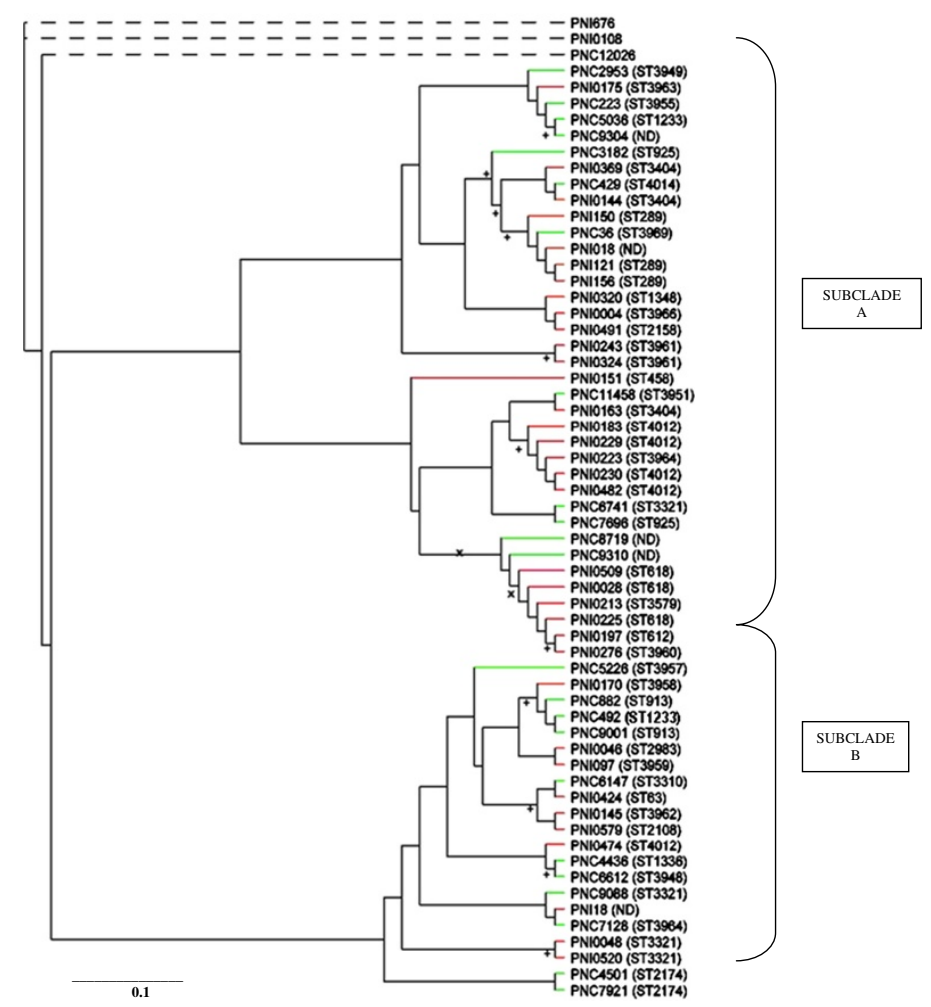

Figure 2 (See legend on next page.) 

are indicated outside brackets; Non-pneumococcal isolates are shown by dotted lines. Invasive isolates are shown in red while carriage isolates are shown in green; + indicates $p=1.0$ while $\times$ indicates $p>0.9$. B: Phylogeny of $S$. pneumoniae isolates (sequence type analysis). Isolate names are shown in brackets while the corresponding MLST are indicated outside brackets; Non-pneumococcal isolates are shown by dotted lines. Invasive isolates are shown in red while carriage isolates are shown in green; STND indicates MLST of the isolate was not determined; + indicates $p=1.0$ while $\times$ indicates $p>0.9$.

the developing world (West Africa), while other studies were based mainly on isolates from developed countries. Comparative phylogenomics of invasive and carriage $S$. pneumoniae isolates identified a number of putative virulence determinants that may be important in the progression of S. pneumoniae from the carriage phase to invasive disease. These putative virulence determinants are currently being investigated by mutagenesis to confirm their role in pneumococcal pathogenicity. Virulence determinants that contribute to S. pneumoniae pathogenicity are likely to be distributed randomly throughout its genome rather than being clustered in dedicated loci or islands. Compared to other S. pneumoniae serotypes, serotype 1 maintains a more uniform genetic content which implies that serotype 1 strains are more likely to be clonally related than strains of other serotypes.

\section{Limitations}

There are a number of limitations of the study. Firstly, the microarray used was based on only two sequenced genomes including TIGR4 and R6 strains, which are reference strains from developed countries rather than the developing world where the study isolates were collected. This means that genes that are absent in the reference strains but present in the study isolates may not be detected. Secondly, it is not known if the genes detected are expressed in vivo or not and if expressed under what conditions. The second limitation is partly addressed by the fact that expressions of some of the virulence genes identified (SP0071, SP0743 and SP1032) have been demonstrated by other investigators [53,54].

\section{Methods}

Identification of S. pneumoniae isolates and extraction of DNA

The study isolates were confirmed to be $S$. pneumoniae by the optochin test [55]. The isolates were purified on $5 \%$ blood agar plates and bacterial chromosomal DNA was prepared using the Wizard gDNA purification kit (Promega). The concentration and purity of extracted DNA was determined by means of a NanoDrop ${ }^{\circledR}$ ND-1000 spectrophotometer (NanoDrop, Wilmington, USA).

\section{Microarray analysis}

S. pneumoniae genomic DNA extracted from the study isolates and reference strain were analysed using the
BuG@S SPv1.1.0 microarray as described previously [43]. This microarray consisted of duplicate spotted PCR products, representing all annotated genes in S. pneumoniae strains TIGR4 and R6. Briefly, $1 \mu \mathrm{g}$ of DNA was labelled by random priming with Klenow polymerase to incorporate either Cy3 or Cy5 dCTP (GE Healthcare) for the reference strain or the test strain, respectively. Equal amounts of the Cy3- and Cy5-labeled samples were copurified through a Qiagen MinElute column (Qiagen), mixed with hybridization solution $(4 \times$ SSC $0.3 \% \mathrm{SDS}$ ), and denatured at $95^{\circ} \mathrm{C}$ for $2 \mathrm{~min}$. The labelled sample was loaded on to a prehybridized microarray under one $22 \mathrm{~mm}$ by $22 \mathrm{~mm}$ Lifter Slip (Erie Scientific), sealed in a humidified hybridization cassette (Corning), and hybridized overnight by immersion in a water bath at $65^{\circ} \mathrm{C}$ for 16 to $20 \mathrm{~h}$. Slides were washed once in $400 \mathrm{ml} 1 \times \mathrm{SSC}, 0.06 \% \mathrm{SDS}$ at $65^{\circ} \mathrm{C}$ for $2 \mathrm{~min}$ and twice in $400 \mathrm{ml} 0.06 \times \mathrm{SSC}$ for $2 \mathrm{~min}$ at room temperature. The microarray slides were then scanned with a GMS 418 Scanner (Genetic Microsystems) and spot fluorescence intensities were determined with ImaGene 5.5 (BioDiscovery Inc.). All the S. pneumoniae study isolates were hybridized once against the TIGR4 reference strain and the microarray hybridization experiments were repeated for isolates which gave poor hybridization results.

\section{Microarray data analysis and comparative phylogenomics} Analysis of the microarray data and comparative phylogenomics were carried out with GeneSpring v6.1 (Silicon Genetics). Data were median normalized in GeneSpring and normalized intensity data for each channel from each microarray were used to run GACK (Genomotypying Analysis Charlie Kim), to determine whether genes were present, absent, or divergent [56]. To run GACK analysis, the raw values were divided by the control values for each sample and then transformed into log2 ratio data. This was saved as a tab delimited file and used as the input file for the GACK software. GACK uses the $\log 2$ ratio data to categorize CDSs based upon estimated probability of presence (EPP). Computation of EPP was done by dividing the mapped normal curve value (the expected value for a distribution in which all spots have signal present on the hybridized microarray) by the actual observed data distribution value for any given ratio [56]. Two stringent cut-offs were used; 
'present' is called only if a GACK EPP was $\geq 100 \%$, 'absent (or highly divergent)' was only called in GACK EPP was $\leq 0 \% \mathrm{EPP}$, 'divergent' genes were between 0 and $100 \%$ EPP. While this cut-off for absent is highly stringent, the stringent hybridisations conditions equate to divergence of greater than approximately $5 \%$ which may result in an 'absent' call to a coding sequence that is present hence 'absent/highly divergent'. The resulting assigned CDS from GACK analysis were re-entered into GeneSpring 6.1 and a core genome of the isolates was determined: core genome was defined as the set of genes present in all the isolates investigated. Genetic differences among the isolates were also determined at a significant level of $\mathrm{p}<0.05$ and Chi square was used to confirm virulence genes (ie genes that were significantly associated with invasive isolates).

The output of GACK was transformed into NEXUS format, and the relationship of the strains was determined based on Bayesian method-based algorithms implemented through Mr Bayes v3.0 software [57]. The resulting phylogenetic trees were viewed using TREEVIEW (http://taxonomy.zoology.gla.ac.uk/rod/treeview. html). Coding sequences (genes) associated with the phylogenomic relationships of isolates and also the formation of clades and subclades were evaluated using MacClade 4 [58].

\section{Ethical considerations}

The study was approved by the ethics committee of the Medical Research Council (The Gambia). The isolates used were gathered from various laboratories and human subjects were not enrolled in the study.

\section{Competing interests}

The authors declare that they have no competing interests.

\section{Authors' contributions}

The study was conceived by BWW, RAS, MA and RAA. Microarray experiments were performed by ESD. Bioinformatics analyses were done by RAS, ESD and JH. The manuscript was drafted and revised by ESD, BWW, RAS, MA, RAA and $\mathrm{JH}$.

\section{Acknowledgements}

We acknowledge the Wellcome trust for funding BuG@S (Bacterial Microarray Group at St. George's, University of London) where microarrays used in the study were obtained. We also acknowledge the Medical Research Council in The Gambia for providing isolates for the study.

\footnotetext{
Author details

'Department of Pathogen Molecular Biology, London School of Hygiene and Tropical Medicine, London WC1E 7HT, UK. ${ }^{2}$ Vaccinology Theme, Medical Research Council Unit, The Gambia. 'Bacterial Microarray Group, St. George's University of London, London SW17 ORE, UK. ${ }^{4}$ GlaxoSmithKline Vaccines, Wavre, Belgium. ${ }^{5}$ Department of Microbiology, University of Ghana Medical School, Accra, Ghana.
}

Received: 30 March 2012 Accepted: 18 October 2012

Published: 29 October 2012

\section{References}

1. Mitchell TJ: Streptococcus pneumoniae: infection, inflammation and disease. Adv Exp Med Biol 2006, 582:111-124.

2. Hill PC, Townend J, Antonio M, Akinsanya B, Ebruke C, Lahai G, et al: Transmission of Streptococcus pneumoniae in rural Gambian villages - a longitudinal study. Clin Infect Dis 2010, 50(11):1468-1476.

3. Sleeman KL, Daniels L, Gardiner M, Griffiths D, Deeks JJ, Dagan R, et al: Acquisition of Streptococcus pneumoniae and nonspecific morbidity in infants and their families: a cohort study. Pediatr Infect Dis J 2005, 24(2):121-127.

4. Black RE, Cousens S, Johnson HL, et al: Global, regional, and national causes of child mortality in 2008: a systematic analysis. Lancet 2010, 375:1969-1987.

5. Rajaratnam JK, Marcus JR, Flaxman AD, et al: Neonatal, postneonatal, childhood, and under-5 mortality for 187 countries, 1970-2010: a systematic analysis of progress towards Millennium Development Goal 4. Lancet 2010, 375:1988-2008.

6. Melin M, Trzciński K, Antonio M, Meri S, Adegbola R, Kaijalainen T, et al: M"pneumoniae. Infect Immun 2010, 78(12):5252-5261.

7. Magee $A D$, Yother J: Requirement for capsule in colonization by Streptococcus pneumoniae. Infect Immun 2001, 69:3755-3761.

8. Adegbola RA, Hill PC, Secka O, Ikumapayi UN, Lahai G, Greenwood BM, Corrah T: Serotype and antimicrobial susceptibility patterns of isolates of Streptococcus pneumoniae causing invasive disease in The Gambia 1996-2003. Trop Med Int Health 2006, 11:1128-1135.

9. Hill PC, Akisanya A, Sankareh K, et al: Nasopharyngeal carriage of Streptococcus pneumoniae in Gambian villagers. Clin Infect Dis 2006 15(6):673-679.

10. Obaro S: Differences in invasive pneumococcal serotypes. Lancet 2001, 357:1800-1801.

11. Brueggemann AB, Spratt BG: Geographic distribution and clonal diversity of Streptococcus pneumoniae serotype 1 isolates. J Clin Microbiol 2003, 41:4966-4970

12. Hausdorff WP: The roles of pneumococcal serotypes 1 and 5 in paediatric invasive disease. Vaccine 2007, 25:2406-2412.

13. Kelly T, Dillard JP, Yother J: Effect of genetic switching of capsular type on virulence of Streptococcus pneumoniae. Infect Immun 1994, 62:1813-1819.

14. Pearson BM, Pin C, Wright Jl, Anson K, Humphrey T, Wells J: Comparative genome analysis of Campylobacter jejuni using whole genome DNA microarrays. FEBS Lett 2003, 554:224-230.

15. Champion OL, Gaunt MW, Gundogdu O, Elmi A, Witney AA, Hinds J, Dorrell $\mathrm{N}$, Wren BW: Comparative phylogenomics of the food-borne pathogen Campylobacter jejuni reveals genetic markers predictive of infection source. Proc Natl Acad Sci USA 2005, 102(44):16043-16048.

16. Howard SL, Gaunt MW, Hinds J, Witney AA, Stabler R, Wren BW: Application of comparative phylogenomics to study the evolution of Yersinia enterocolitica and to identify genetic differences relating to pathogenicity. J Bacteriol 2006, 188(10):3645-3653.

17. Stabler RA, Gerding DN, Songer JG, Drudy D, Brazier JS, Trinh HT, Witney AA, Hinds J, Wren BW: Comparative phylogenomics of Clostridium difficile reveals clade specificity and microevolution of hypervirulent strains. J Bacteriol 2006, 188(20):7297-7305.

18. Howard SL, Jagannathan A, Soo EC, Hui JP, Aubry AJ, Ahmed I, Karlyshev A, Kelly JF, Jones MA, Stevens MP, Logan SM, Wren BW: Campylobacter jejuni glycosylation island important in cell charge, legionaminic acid biosynthesis, and colonization of chickens. Infect Immun 2009, 77(6):2544-2556.

19. Hakenbeck R, Balmelle N, Weber B, Gardes C, Keck W, De Saizieu A: Mosaic genes and mosaic chromosomes: intra- and interspecies genomic variation of Streptococcus pneumoniae. Infect Immun 2001, 69:2477-2486.

20. Tettelin H, Nelson KE, Paulsen IT, Eisen JA, Read TD, Peterson S, et al: Complete genome sequence of a virulent isolate of Streptococcus pneumoniae. Science 2001, 293:498-506.

21. Obert C, Sublett J, Kaushal D, Hinojosa E, Barton T, Tuomanen El, Orihuela CJ: Identification of a Candidate Streptococcus pneumoniae core genome and regions of diversity correlated with invasive pneumococcal disease. Infect Immun 2006, 74:4766-4777.

22. Hiller NL, Janto B, Hogg JS, Boissy R, Yu S, Powell E, et al: Comparative genomic analyses of seventeen Streptococcus pneumoniae strains: insights into the pneumococcal supragenome. J Bacteriol 2007, 189:8186-8195. 
23. Donati C, Hiller NL, Tettelin H, Muzzi A, Croucher NJ, Angiuoli SV, et al: Structure and dynamics of the pan-genome of Streptococcus pneumoniae and closely related species. Genome Biol 2010, 11(10):R107.

24. Slotved HC, Kaltoft M, Skovsted IC, Kerrn MB, Espersen F: Simple, rapid latex agglutination test for serotyping of pneumococci (PneumotestLatex). J Clin Microbiol 2004, 42:2518-2522.

25. Enright MC, Spratt BG: A multilocus sequence typing scheme for Streptococcus pneumoniae: identification of clones associated with serious invasive disease. Microbiology 1998, 144(11):3049-3060.

26. Hill PC, Akisanya A, Sankareh K, Cheung YB, Saaka M, Lahai G, Greenwood BM, Adegbola RA: Nasopharyngeal carriage of Streptococcus pneumoniae in Gambian villagers. Clin Infect Dis 2006, 43(6):673-679.

27. Falade AG, Lagunju IA, Bakare RA, Odekanmi AA, Adegbola RA: Invasive pneumococcal disease in children aged $<5$ years admitted to 3 urban hospitals in Ibadan, Nigeria. Clin Infect Dis 2009, 48(2):190-196.

28. Donkor ES, Newman MJ, Oliver-Commey J, Bannerman E, Dayie NTKD, Badoe EV: Invasive disease and paediatric carriage of Streptococcus pneumoniae in Ghana. Scand J Infect Dis 2010, 42:254-259.

29. Hill PC, Cheung YB, Akisanya A, Sankareh K, Lahai G, Greenwood BM Adegbola RA: Nasopharyngeal carriage of Streptococcus pneumoniae in Gambian infants: a longitudinal study. Clin Infect Dis 2008, 46(6):807-814

30. Rasmussen TB, Danielsen M, Valina O, Garrigues C, Johansen E, Pedersen MB: Streptococcus thermophilus core genome: comparative genome hybridization study of 47 strains. Appl Environ Microbiol 2008, 74:4703-4710.

31. Lang P, Lefebure T, Wang W, Zadoks RN, Schukken Y, Stanhope MJ: Gene content differences across strains of Streptococcus uberis identified using oligonucleotide microarray comparative genomic hybridization. Infect Genet Evol 2009, 9:179-188.

32. Tettelin H, Masignani V, Cieslewicz MJ, Eisen JA, Peterson S, Wessels MR, et al: Complete genome sequence and comparative genomic analysis of an emerging human pathogen, serotype V Streptococcus agalactiae. Proc Natl Acad Sci USA 2002, 99:12391-12396.

33. Hoskins J, Alborn WE, Arnold J, Blaszczak LC, Burgett S, Dehoff BS, et al: Genome of the bacterium Streptococcus pneumoniae strain R6. J Bacteriol 2001, 183:5709-5717.

34. Dopazo J, Mendoza A, Herrero J, Caldara F, Humbert Y, et al: Annotated draft genomic sequence from Streptococcus pneumoniae type $19 \mathrm{~F}$ clinical isolate. Microb Drug Resist 2001, 7:99-125.

35. Camilli R, Bonnal RJ, Del Grosso M, lacono M, Corti G, et al: Complete genome sequence of a serotype 11A, ST62 Streptococcus pneumoniae invasive isolate. BMC Microbiol 2011, 11:25.

36. Lanie JA, Ng WL, Kazmierczak KM, Andrzejewski TM, Davidsen TM, Wayne KJ, Tettelin H, Glass JI, Winkler ME: Genome sequence of Avery's virulent serotype 2 strain D39 of Streptococcus pneumoniae and comparison with that of unencapsulated laboratory strain R6. J Bacterio/ 2007, 189:38-51.

37. Ding F, Tang P, Hsu MH, Cui P, Hu S, Yu J, Chiu CH: Genome evolution driven by host adaptations results in a more virulent and antimicrobial resistant Streptococcus pneumoniae serotype 14. BMC Genomics 2009, 10:158.

38. Gilliespie SH, Balakrishnan I: Pathogenesis of pneumococcal infection. J Med Microbiol 2000, 49:1057-1067.

39. Preston JA, Dockrell DH: Virulence factors in pneumococcal respiratory pathogenesis. Future Microbiol 2008, 3(2):205-221.

40. Garmory HS, Titball RW: ATP-binding cassette transporters are targets for the development of antibacterial vaccines and therapies. Infect Immun 2004, 72:6757-6763

41. Hava DL, Camilli A: Large-scale identification of serotype 4 Streptococcus pneumoniae virulence factors. Mol Microbiol 2002, 45:1389-1406.

42. Blomberg C, Dagerhamn J, Dahlberg S, Browall S, Fernebro J, Albiger B, et al: Pattern of accessory regions and invasive disease potential in Streptococcus pneumoniae. J Infect Dis 2009, 199(7):1032-1042.

43. Silva NA, McCluskey J, Jefferies JM, Hinds J, Smith A, Clarke SC, Mitchell TJ, Paterson GK: Genomic diversity between strains of the same serotype and multilocus sequence type among pneumococcal clinical isolates. Infect Immun 2006, 74(6):3513-3518.

44. Brueggemann AB, Griffiths DT, Meats E, Peto T, Crook DW, Spratt BG: Clonal relationships between invasive and carriage Streptococcus pneumoniae and serotype- and clone-specific differences in invasive disease potential. J Infect Dis 2003, 187:1424-1432.
45. Brueggemann $A B$, Pai $R$, Crook DW, Beall B: Vaccine escape recombinants emerge after pneumococcal vaccination in the United States. PLOS Pathog 2007, 3(11):e168.

46. Dobrindt U, Blum-Oehler G, Nagy G, Schneider G, Johann A, Gottschalk G, Hacker J: Genetic structure and distribution of four pathogenicity islands PAI I(536) to PAI IV (536) of uropathogenic Escherichia coli strain 536. Infect Immun 2002, 70(11):6365-6372.

47. Dagerhamn J, Blomberg C, Browall S, Sjostrom K, Morfeldt E, Henriquesnormark B: Determination of accessory gene patterns predicts the same relatedness among strains of Streptococcus pneumoniae as sequencing of housekeeping genes does and represents a novel approach in molecular epidemiology. J Clin Microbio/ 2008, 46:863-868.

48. Turner P, Hinds J, Turner C, Jankhot A, Gould K, Bentley SD, et al: Improved detection of nasopharyngeal co-colonization by multiple pneumococcal serotypes by use of latex agglutination or molecular serotyping by microarray. J Clin Microbiol 2011, 49(5):1784-1789.

49. Croucher NJ, Harris SR, Fraser C, Quail MA, Burton J, van der Linden M, et al: Rapid pneumococcal evolution in response to clinical interventions. Science 2011, 331(6016):430-434.

50. Feil EJ, Smith JM, Enright MC, Spratt BG: Estimating recombinational parameters in Streptococcus pneumoniae from multilocus sequence typing data. Genetics 2000, 154:1439-1450.

51. Embry A, Hinojosa E, Orihuela CJ: Regions of Diversity 8,9 and 13 contribute to Streptococcus pneumoniae virulence. BMC Microbio/ 2007 7:80.

52. McAllister $L J$, Ogunniyi AD, Stroeher UH, Paton JC: Contribution of a Genomic Accessory Region Encoding a Putative Cellobiose Phosphotransferase System to Virulence of Streptococcus pneumoniae. PLoS One 2012, 7(2):e32385.

53. Polissi A, Pontiggia A, Feger $G$, Altieri M, Mottl H, Ferrari L, et al: Large-scale identification of virulence genes from Streptococcus pneumoniae. Infect Immun 1998, 66:5620-5629.

54. Rogers PD, Liu TT, Barker KS, Hilliard GM, English BK, Thornton J, et al: Gene expression profiling of the response of Streptococcus pneumoniae to penicillin. J Antimicrob Chemother 2007, 59:616-626.

55. Bowers EF, Jeffries LR: Optochin in the identification of str. pneumoniae. J Clin Pathol 1955, 8:58-60.

56. Kim CC, Joyce EA, Chan K, Falkow S: Improved analytical methods for microarray-based genome-composition analysis. Genome Biol 2002, 3:1-17.

57. Ronquist F, Huelsenbeck JP: MrBayes 3: Bayesian phylogenetic inference under mixed models. Bioinformatics 2003, 19:1572-1574.

58. Maddison DR, Maddison WP: MacClade 4: analysis of phylogeny and character evolution. Sunderland, Mass: Sinauer Associates; 2001. Version 4.03.

doi:10.1186/1471-2164-13-569

Cite this article as: Donkor et al:: Comparative phylogenomics of Streptococcus pneumoniae isolated from invasive disease and nasopharyngeal carriage from West Africans. BMC Genomics 2012 13:569.

\section{Submit your next manuscript to BioMed Central and take full advantage of:}

- Convenient online submission

- Thorough peer review

- No space constraints or color figure charges

- Immediate publication on acceptance

- Inclusion in PubMed, CAS, Scopus and Google Scholar

- Research which is freely available for redistribution 